Péter Maitz.

\title{
Wohin steuert die Historische Sprachwissenschaft? Erkenntniswege und Profile einer scientific community im Wandel
}

\section{Problemstellung}

Dieses Buch handelt von der Historischen Sprachwissenschaft, ihren Erkenntnisinteressen, Grundlagenproblemen und Desideraten. Die Fragestellungen der einzelnen Beiträge sind dementsprechend zumeist durch metawissenschaftliche ${ }^{1}$ Probleme motiviert. Die Autorinnen und Autoren ${ }^{2}$ reflektieren - in der Regel gestützt durch objektwissenschaftliche Analysen - Möglichkeiten und Grenzen der unterschiedlichen Erkenntniswege, theoretische und methodologische Grundsatzfragen, nicht zuletzt aber auch wissenschaftsgeschichtliche, erkenntnistheoretische, wissenssoziologische Aspekte der Forschung.

Wenn dabei von ,Historischer Sprachwissenschaft ${ }^{6}$ die Rede ist, so wird der Begriff - den Entwicklungen der letzten Jahrzehnte Rechnung tragend und im Gegensatz zu anderen, engeren Auslegungen (vgl. z. B. Mattheier 1998: 824 oder Polenz 2000: 9) - im weitestmöglichen Sinne verwendet. Es wird darunter diejenige linguistische Forschung verstanden, die ihren Gegenstand, die ,Sprache', wie auch immer sie konzeptualisiert werden mag, als historisch gewachsenes und sich wandelndes Phänomen begreift und sie im Zusammenhang damit - entweder aus historischsynchroner, diachroner oder synchron-variationslinguistischer Perspektive - auf ihre Geschichte bzw. ihren Wandel hin untersucht, beschreibt und erklärt.

Eine solche metawissenschaftliche Perspektive auf die Historische Sprachwissenschaft scheint mindestens aus drei Gründen vielverspre-

1 Unter ,Metawissenschaft ${ }^{\star}$ wird - im Gegensatz zu ,Objektwissenschaft ${ }^{\star}$ - eine Wissenschaft verstanden, deren Gegenstand selbst eine Wissenschaft ist.

2 Aus platzökonomischen Gründen verwende ich im Folgenden bei Bezeichnungen für Personen nur das generische Maskulinum. 
chend, vielleicht sogar notwendig zu sein. Zum einen kann die bewusste und kritische Auseinandersetzung mit den in der alltäglichen Forschungspraxis oft unhinterfragt befolgten Forschungsnormen maßgeblich auch zur Lösung objektwissenschaftlicher Probleme beitragen. Zum anderen können auf diese Weise ebenso grundlegende theoretische und methodologische Desiderate sichtbar gemacht werden. Und schließlich kann die Reflexion über die interne Dynamik der Historischen Sprachwissenschaft auch wissenschaftstheoretisch bzw. epistemologisch relevante Erkenntnisse ans Tageslicht fördern, aus denen sich jedoch keineswegs nur für die Wissenschafts- und Erkenntnistheorie, sondern durchaus auch für die Historische Sprachwissenschaft selbst wichtige Konsequenzen ergeben können. Innerhalb der Historischen Sprachwissenschaft - als Forschungsgebiet wie auch als wissenschaftliche Gemeinschaft - spielten sich nämlich in den vergangenen Jahrzehnten Entwicklungen ab, die zum einen aus wissenschaftstheoretischer Sicht bemerkenswert sind, zum anderen aber auch für den objektwissenschaftlichen Erkenntnisfortschritt innerhalb der Disziplin gewichtige Konsequenzen haben. Im Folgenden sollen zunächst - allein schon aus Umfangsgründen in stark vereinfachter und rudimentärer Form - diese Entwicklungen kurz nachgezeichnet werden. ${ }^{3}$

\section{Die Anfänge}

Die Geschichte der Historischen Sprachwissenschaft ließe sich in drei wohlunterschiedene, voneinander recht klar abgrenzbare Phasen einteilen, wenn man für diese Einteilung zwei Kriterien heranzieht: (a) die Grundlagenstabilität, d. h. die Konsenshaftigkeit der forschungsleitenden Werte und Normen sowie (b) die Schärfe der Gruppengrenze, die die wissenschaftliche Gemeinschaft von der wissenschaftlichen Außenwelt trennt.

Die erste von diesen drei Phasen umfasst jene Periode, in der die historische Sprachforschung im Grunde noch als die einzig denkbare und legitime Form der wissenschaftlichen Beschäftigung mit Sprache gilt. Diese Periode, das 19. Jh. also, ist durch eine - zumindest im Vergleich zum gegenwärtigen Profil der Forschungslandschaft auffallende - relative Grundlagenstabilität gekennzeichnet. Vor dem Hintergrund eines positivistischen Wissenschaftsverständnisses ${ }^{4}$ dominieren (radikal) induktivis-

3 Die nachfolgenden Ausführungen basieren auf Maitz (i. Vorb.).

4 Der (auch) im Kontext der Historischen Sprachwissenschaft stark negativ geladene und zumeist als pauschales Schimpfwort verwendete Terminus ,Positivismus' wird hier selbstverständlich im wertneutralen Sinne verwendet. Darunter wird die Abneigung gegen eine explizite Theoriebildung verstanden, die Auffassung also, dass es angesichts der vermeint- 
tisch betriebene Forschungen zum germanischen Altertum und zur älteren deutschen Sprachgeschichte. Es handelt sich also um eine kumulative Forschungsphase mit allgemein anerkannten und konsenshaft befolgten (historisch-komparatistischen und später junggrammatischen) Forschungsnormen. Die weitgehende Dominanz der zeitgenössischen Historischen Sprachwissenschaft innerhalb der Linguistik, die starke interne Kohäsion der wissenschaftlichen Gemeinschaft und die Autorität ihrer Vertreter machen es möglich, dass konkurrierende Erkenntniswege und wissenschaftliche Leistungen vielfach abgelehnt oder einfach ignoriert werden und bestenfalls ein Schattendasein führen können. Dieser Situation, d. h. der starken Machtposition und Verschlossenheit der zeitgenössischen Historischen Sprachwissenschaft fällt u. a. auch Humboldts Schaffen zum Opfer. Humboldts Schüler, Heymann Steinthal stellt fest:

Humboldt hat als Sprachforscher auf keinen seiner älteren oder jüngeren Zeitgenossen in spezifischer Weise, d. h. durch die ihm eigenthümlich angehörenden, von ihm geschaffenen Ideen eingewirkt. Er hat wohl von den Schlegels, den Grimms, den Bopps gelernt, sie aber von ihm durchaus nicht. (zit. nach Arens 1974: 205)

Und nach einer eingehenden Analyse des zeitgenössischen Rezensionswesens sieht auch Storost (1985) diese Behauptung bestätigt, indem er konstatiert:

[D]ie sprachphilosophischen Gedankengänge Humboldts werden in keiner Weise nachvollzogen, mitgedacht, mitgeteilt, kommentiert, kritisiert oder auch nur angedeutet. (Storost 1985: 323)

Diese erste, progressive Phase der Geschichte der Historischen Sprachwissenschaft ist also durch eine relative Grundlagenstabilität in der Forschung und eine die linguistische Forschungslandschaft dominierende scientific community mit scharfer Gruppengrenze und starker interner Kohäsion gekennzeichnet. Grundlegend abweichende Positionen werden - wie der Fall von Humboldt oder später von Georg von der Gabelentz zeigt nicht akzeptiert. Die historisch-philologische Arbeitsweise ist und bleibt zunächst weitgehend dominant, sowohl ahistorische als auch hypothetisch-deduktive bzw. sprachphilosophische Herangehensweisen werden vielfach als inadäquat und spekulativ abgestempelt und bewusst unterdrückt (vgl. Schmidt 1985: 169f.).

Nach diesem progressiven ersten Abschnitt bricht ab der ersten Hälfte des 20. Jh. eine zweite, stagnative Phase ein. Durch das Aufkommen und

lichen Selbstevidenz des Gegenstandes genügen würde, ohne Hypothesen an das Material heranzugehen (vgl. Boretzky 1977: 33). 
die Verbreitung ahistorischer Forschungsrichtungen in der Linguistik wird die Historische Sprachwissenschaft nunmehr in eine Art Defensive gedrängt. Zumindest gilt dies für den Status und das Prestige historischer Forschungen. Während es im 19. Jh. noch die Historische Sprachwissenschaft war, die Humboldts sprachphilosophisches Werk als spekulativ abstempeln und ahistorische Forschungen an die Peripherie der Linguistik drängen konnte, so hat sich diese Situation im Laufe des 20. Jh. umgekehrt. Zunächst vom Strukturalismus und später von der Generativen Grammatik wurde die Position der Historischen Sprachwissenschaft zutiefst erschüttert; es wurde die linguistische Relevanz historischer Beschreibungen und genetischer Erklärungen grundsätzlich in Frage gestellt und der gesamten historischen Sprachforschung ein erhebliches Theoriedefizit vorgeworfen. Die veränderten Prestigeverhältnisse zeigt in sehr transparenter Weise folgendes Zitat:

Es genügt nicht, das Ohr an die Daten zu legen, bzw. die Daten allein sind stumm. [...] Es darf als Verdienst der Generativen Grammatik betrachtet werden, daß sie die Problematik der reinen (in der traditionellen historischen Sprachwissenschaft nicht unüblichen) Datenhuberei erneut thematisiert hat. Viele historische Linguisten erschöpft die (gewiß notwendige, unverzichtbare und ermüdende) Dateneinfuhr dermaßen, daß sie vor der eigentlichen (interpretativ-theoretischen) Arbeit aufhören. Die Sensitiveren unter ihnen haben seit der Generativen Grammatik wenigstens ein schlechtes Gewissen. (Mayerthaler 1998: 530) ${ }^{5}$

In wissenschaftssoziologischer Hinsicht ist es jedoch bemerkenswert, dass die - nach wie vor strikt philologisch orientierte - Historische Sprachwissenschaft trotz ihres Prestigeverlusts zunächst noch ihre starke institutionelle Position beibehalten kann. Germanistische Lehrstühle sind zumeist auch noch in den Nachkriegsjahrzehnten der ungeteilten, historisch orientierten germanischen Philologie (der ,älteren deutschen Sprache und Literatur") gewidmet. Und ebenfalls erst in dieser Zeit, in den 1960er und 1970er Jahren, kommt es zur Gründung der ersten germanistischen Fachzeitschriften, die bereits der ,modernen', nicht mehr der philologischhistorischen Tradition verpflichteten Linguistik ein Forum bieten sollen.

Was den Inhalt und vor allem die sprachtheoretischen und forschungsmethodologischen Grundlagen der historischen Sprachforschung betrifft, so ändert sich zunächst ebenfalls nicht viel. Die auch für die erste, progressive wissenschaftsgeschichtliche Phase kennzeichnende Grundla-

5 Zwar stammt dieses Zitat bereits aus der dritten, unten näher zu erläuternden wissenschaftsgeschichtlichen Phase der Historischen Sprachwissenschaft. Doch seine Grundhaltung und seine Aussage stehen eindeutig in der Tradition jener von außen kommenden Angriffe, die sich spätestens seit dem Auftreten des linguistischen Strukturalismus schon ab der ersten Hälfte des 20. Jh. - wenn auch zunächst in weniger polemischer Weise melden. 
genstabilität bleibt erhalten. ${ }^{6}$ Die Historische Sprachwissenschaft beharrt auf den Forschungsnormen, die aus dem 19. Jh. ererbt worden sind. Fragestellungen und Forschungsmethoden folgen weitgehend der junggrammatischen Tradition, sie bleiben über Generationen hinweg ,relativ konstant und unbestritten“ (Gardt/Mattheier/Reichmann 1995: VII). Zur Erhaltung dieser Grundlagenstabilität trägt selbstverständlich auch die inzwischen ausgesprochen feindselig gewordene Umgebung maßgeblich bei. Die Vertreter der neu aufkommenden, zumeist strikt ahistorischen Forschungsrichtungen fordern die Historische Sprachwissenschaft, wie das obige Zitat zeigt, vielfach auf eine ausgesprochen provokative und aggressive Weise heraus. Diese äußere Bedrohung sowie die scheinbare Unversöhnlichkeit der historisch-junggrammatischen Arbeitsweise mit den neuen Forschungsansätzen führen dazu, dass sich die Historische Sprachwissenschaft - nach wie vor - nach außen verschließt, was sie sich aufgrund ihrer starken und gefestigten institutionellen Einbettung (zunächst noch) leisten kann.

Zusammenfassend kann man also in dieser zweiten wissenschaftsgeschichtlichen Phase der Historischen Sprachwissenschaft - ähnlich zur ersten - von einer institutionell gefestigten und ihre Machtposition zunächst bewahrenden scientific community sprechen. Ihre starke interne Organisation und ihre scharfe Gruppengrenze bleiben im Grunde ebenso bestehen wie die Grundlagenstabilität der Forschungspraxis, und zwar trotz des Umstands, dass die historisch-philologischen Analyseverfahren - im Gegensatz zur ersten Phase - einen nicht unerheblichen Prestigeverlust erfahren und von außen grundsätzlich in Frage gestellt werden.

\section{Der Umbruch}

Die oben beschriebene Situation ändert sich ab den 1970er Jahren grundsätzlich. $\mathrm{Zu}$ dieser Zeit setzen wissenschaftsinterne und auch wissenschaftssoziologische Entwicklungen ein, die bis heute andauern und das Profil der Historischen Sprachwissenschaft maßgeblich prägen.

$\mathrm{Ab}$ dieser Zeit tritt die Historische Sprachwissenschaft aus der stagnativen zweiten Phase ihrer Geschichte in eine progressive, auch die Gegen-

6 Freilich melden sich konkurrierende Forschungsansätze auch in dieser Phase. So etwa um nur ein einziges Beispiel zu nennen - die Prager Strukturalisten, die mit dem Begriff der ,dynamischen Synchronie' und dem Prinzip der ,strukturellen Diachronie‘ die Saussure'sche Kluft zwischen synchroner und diachroner Sprachwissenschaft zu überbrücken versuchen und u. a. die Historische Phonologie begründen (Jakobson 1931). Doch derartige Ansätze gefährden (zunächst) keineswegs das Prestige und die Dominanz der junggrammatischen Arbeitsweise innerhalb der scientific community. 
wart mit einschließende dritte. Als progressiv kann diese Phase in zweifacher Hinsicht bezeichnet werden. Zum einen öffnet sich ein bedeutender Teil der bis dahin für historische Fragestellungen verschlossenen und vielfach auch feindlichen linguistischen Welt außerhalb der Historischen Sprachwissenschaft für die Historizität und die Dynamik von Sprache (vgl. Cherubim 1975: 2f., Boretzky 1977: 11). Zum anderen werden die klassisch-junggrammatischen Grundlagen sprachhistorischer Forschungen unter externem Einfluss - auch innerhalb der Historischen Sprachwissenschaft selbst immer öfter und stärker in Frage gestellt. Auch die Historische Sprachwissenschaft selbst öffnet sich also immer mehr für neue theoretische und methodologische Zugangsweisen. Durch diese beiden parallelen Entwicklungen wird die scharfe Grenze, die die wissenschaftliche Gemeinschaft von der linguistischen Außenwelt bis dahin getrennt hat, in einem bis dahin nie gesehenem Maße aufgelockert.

$\mathrm{Zu}$ dieser immer intensiveren Annäherung einst antagonistisch erscheinender Positionen tragen natürlich zahlreiche Faktoren bei. Obwohl der Zeitraum bzw. die Entwicklungen, von denen hier die Rede ist, bislang keinen systematischen wissenschaftsgeschichtlichen Analysen unterzogen worden sind, so sollen manche der wichtigsten Faktoren, die in diesem Zusammenhang von Belang gewesen sein dürften, zumindest angedeutet werden.

Von entscheidender Bedeutung ist erstens der Umstand, dass die progressiven linguistischen Schulen, die die historisch-dynamische Sprachbetrachtung in die Defensive gedrängt hatten, besonders ab den 1960er Jahren von verschiedenen Seiten und auf verschiedenen Ebenen heftig angegriffen wurden. Zum einen ist mehrfach auf die Unhaltbarkeit des strukturalistischen Postulats homogener Sprachsysteme und der scharfen Trennung zwischen Synchronie und Diachronie hingewiesen worden (vgl. z. B. Coseriu 1974, Weinreich/Labov/Herzog 1968 etc.). Im Zuge dessen kam es auch zur Begründung oppositioneller, dynamischer, die Kluft zwischen Synchronie und Diachronie überbrückender Sprachtheorien, wie etwa der Soziolinguistik (vgl. etwa Weinreich/Labov/Herzog 1968), der Natürlichkeitstheorie (vgl. Mayerthaler 1981, Wurzel 1984) oder der Grammatikalisierungstheorie (vgl. Lehmann 1995). Zum anderen wurden ab dieser Zeit genauso auch die sprachtheoretischen und methodologischen Grundlagen der Generativen Grammatik heftig angegriffen und es wurde auch deren wissenschaftsgeschichtliche Bedeutung angezweifelt. Besonders ab den 1970er Jahren meldeten sich immer häufiger wissenschaftsgeschichtliche Interpretationen (z. B. Anttila 1975, Gray 1976, Murray 1980), die der gängigen Meinung, wonach Chomskys Auftreten in der Linguistik eine wissenschaftliche Revolution im Kuhn'schen Sinne eingeleitet hätte, skeptisch gegenüberstanden. Statt einer chomskyanischen 
Revolution sprachen sie von einem erfolgreichen Putsch, der von einer militanten Gruppe von Linguisten und begleitet von einer effektiven revolutionären Rhetorik mit dem Ziel der Machtergreifung durchgeführt wurde (vgl. Kertész 2009: 397). Auf diese Weise, durch die immer lauter werdenden kritischen Stimmen und das Aufkommen von progressiven Konkurrenten kam es dazu, dass die Position bzw. das Prestige der Richtungen geschwächt wurde, die der Historischen Sprachwissenschaft feindlich gegenüberstanden.

Zweitens haben diese konkurrierenden Schulen nicht nur die (einstigen) ,Feinde historisch orientierter Forschungen angegriffen, sondern sie boten der Historischen Sprachwissenschaft zugleich auch die Möglichkeit an, an progressive Tendenzen in der Linguistik anzuknüpfen und auf diese Weise aus der stagnativen Phase herauszukommen. Im Anschluss daran wurden ab den 1970er und 1980er Jahren (Teil)Disziplinen wie - um hier nur zwei bis heute prominente von ihnen zu nennen - die Historische Pragmatik (vgl. z. B. Sitta 1980) und die Historische Soziolinguistik (vgl. z. B. Romaine 1982) begründet und ausgebaut. Bemerkenswert ist allerdings die Tatsache, dass sich diese neuen Richtungen innerhalb der Historischen Sprachwissenschaft selbstständig, d. h. unabhängig von ihren ahistorisch-synchronen Pendants etabliert und institutionalisiert hatten. ${ }^{7}$ Die scharfe Grenze zwischen Synchronie und Diachronie, zwischen gegenwartsbezogener und historisch orientierter Sprachwissenschaft ist also bestenfalls sprachtheoretisch und methodologisch, keineswegs aber im soziologischen bzw. institutionellen Sinne aufgelockert bzw. aufgehoben worden. Durch die eigenständige Etablierung der Historischen Pragmatik, Soziolinguistik oder Semantik wurde sie sogar institutionalisiert und damit weiter verfestigt.

In diesem Sinne hat sich die Gruppengrenze, die die Historische Sprachwissenschaft von anderen wissenschaftlichen Gemeinschaften innerhalb der Linguistik trennt, zwar ohne Zweifel aufgelockert, sie ist aber keineswegs verschwunden. Dies ist jedoch zumindest unter soziologischem Aspekt auch nicht verwunderlich. Denn die Aufhebung der Gruppengrenze und die Integration einst getrennter wissenschaftlicher Gemeinschaften hätten einen verstärkten Konkurrenzkampf als notwendige Konsequenz gehabt und zugleich auch die institutionelle Autonomie der Historischen Sprachwissenschaft gefährdet.

7 So haben auch die genannten beiden Disziplinen ihre eigenen Organisationen, Konferenzen und Publikationsforen ins Leben gerufen; man denke nur an die Gründung des Journal of Historical Pragmatics neben dem Journal of Pragmatics, an das Historical Sociolinguistics Network, die Internet-Zeitschrift Historical Sociolinguistics and Sociobistorical Linguistics oder die Tagungsreihe Historische Soziolinguistike des Deutschen. 
Konvergenz und Integration - wie auch Divergenz - sind in der Forschungspraxis oder zwischen wissenschaftlichen Gemeinschaften aber keineswegs rein rationale Phänomene. In derartigen Dynamiken spielen oft auch psychologische Faktoren eine entscheidende Rolle (vgl. Kuhn 1976, Barnes/Bloor/Henry 1996). So war zur Öffnung der Historischen Sprachwissenschaft drittens auch die Entstehung und Verbreitung eines Krisenbewusstseins notwendig, das die Öffnung der Disziplin für neue Zugangsweisen als notwendig, zumindest aber als vorteilhaft erscheinen ließ. Dass dieses Krisenbewusstsein tatsächlich und zwar schon über einen längeren Zeitraum hinweg vorhanden war, belegt folgendes Zitat:

Beschäftigt man sich in neuerer Zeit mit der Sprachgeschichte des Deutschen, so gehört es fast schon zum guten Ton, ein Lamento abzustimmen über den Verfall sprachhistorischer Traditionen in der Wissenschaftsgeschichte der Linguistik, über die Lückenhaftigkeit des Gegenstandsspektrums der Sprachgeschichte und über fehlende methodologische Konzepte. Während Sonderegger noch zurückhaltend von der ,Problematik der deutschen Sprachgeschichtsschreibung' spricht, werden von Cherubim und anderen lange Listen von Desideraten und Forschungslücken zusammengestellt. (Mattheier 1995: 1)

Die Erneuerung einer Disziplin, die Veränderung der Forschungsnormen innerhalb einer wissenschaftlichen Gemeinschaft kann nur erfolgen, wenn die Mitglieder dieser Gemeinschaft auch individuell an der Erneuerung und der Übernahme der neuen Normen interessiert sind. Als vierter Faktor muss auch die Rolle dieser individuellen Ebene erwähnt und damit also danach gefragt werden, inwiefern die Öffnung etwa in Richtung der Soziolinguistik, der Pragmatik oder der Sprachtypologie auch für den einzelnen Forscher als verheißungsvoll erscheinen konnte. Zum einen darf nicht übersehen werden, dass es sich bei all den genannten Forschungsrichtungen um progressive Schulen handelt, die - auch außerhalb der Historischen Sprachwissenschaft - als prestigevoll und neuartig genug galten, um die neueren Forschungsgenerationen anzuziehen. Zum anderen waren sie offen genug, um diesen Forschergenerationen zahlreiche als relevant anerkannte ungelöste Probleme zu bieten, nicht zuletzt allein schon dadurch, dass sie auch für historische bzw. diachrone Fragestellungen of fen waren. Somit haben sie über beide grundlegenden Eigenschaften verfügt, die nach Kuhn (1976: 25) Kandidaten für wissenschaftliche Paradigmen auszeichnen. Und drittens dürfte auch in unserem Zusammenhang noch ein weiterer Faktor von Belang gewesen sein, den Schmidt (1988) im Kontext der Etablierung des Strukturalismus in Deutschland erwähnt. Die neuen Forschergenerationen dürften demnach auch insofern am Bruch mit der philologisch-junggrammatischen Forschungstradition und an dem Anschluss an gruppenexterne, neue Forschungsnormen interessiert gewe- 
sen sein, als es ihnen schnellen Erfolg versprach, indem es erlaubte, „sich all das immense Wissen, das in hundert Jahren Sprachgeschichtsforschung zusammengetragen worden war, nicht aneignen zu müssen“ (Schmidt 1988; Hervorhebung im Original).

Neben all den genannten werden sicher auch noch weitere, durch zukünftige wissenschaftsgeschichtliche Analysen zu ermittelnde Faktoren dazu beigetragen haben, dass sich die Historische Sprachwissenschaft ab den 1970er Jahren sowohl inhaltlich als auch vom Profil der Gemeinschaft her grundlegend verändert hat. Im Sinne des Gesagten wurde die einst scharfe Gruppengrenze deutlich aufgelockert und die frühere Grundlagenstabilität der Forschung wurde durch einen ausgeprägten Pluralismus abgelöst. Die Entwicklungen der letzten Jahrzehnte und auch die Gegenwart sind von einer nie vorher gesehenen Grundlageninstabilität und Diversifikation innerhalb des Faches, von einem Wettbewerb zwischen zahlreichen theoretisch und methodologisch divergierenden Forschungsansätzen gekennzeichnet. Induktivistische Zugangsweisen (z. B. Historische Philologie, junggrammatisch geprägte Historische Grammatik, Soziopragmatische Sprachgeschichte) existieren heute neben hypothetisch-deduktiv ausgerichteten Forschungen (z. B. Grammatikalisierungsforschung, Diachrone Sprachtypologie, Sprachwandeltheorie); systemimmanente (z. B. strukturalistische, typologische) Beschreibungs- und Erklärungsansätze neben stark gesellschafts- und kulturorientierten (wie z. B. Historische Pragmatik und Soziolinguistik); stark interdisziplinär angelegte Ansätze (z. B. Diskursgeschichte oder Kulturgeschichte der Sprache) neben eher reduktionistischen (z. B. Historische Grammatik).

\section{Paradigmen in der Historischen Sprachwissenschaft?}

Die theoretischen und methodologischen Grundlagen und somit auch die Gegenstände vieler dieser neueren Forschungsrichtungen divergieren in dem Maße, dass es auf den ersten Blick gar nicht überrascht, wenn mehrere Forscher bereits von unterschiedlichen wissenschaftlichen Paradigmen innerhalb der Historischen Sprachwissenschaft sprechen: Reichmann (1998: 6) vom historisch-genetischen Paradigma, Ágel (1999: 180) vom Grammatikalisierungsparadigma, Scharloth (2005) vom soziopragmatischen Paradigma, Greule (2005: 19) sogar vom Forschungsparadigma „Kanzleisprachen“ - und die Reihe ließe sich weiter fortsetzen. Es ist allerdings kaum zu übersehen, dass der Begriff ,Paradigma“ hier von verschiedenen Autoren zum Teil mit unterschiedlicher Intension verwendet wird. In vielen dieser Fälle handelt es sich um eine - auch außerhalb der Linguistik weit verbreitete - theorieneutrale Verwendung, so dass mit 
,Paradigma' nichts weiter als ein relativ klar abgrenzbarer Forschungsansatz bzw. eine wissenschaftliche Schule gemeint wird. In anderen Fällen geschieht die Verwendung des Begriffs ,Paradigma' bereits unter expliziter Bezugnahme auf Thomas S. Kuhns Wissenschaftstheorie (Kuhn 1976), in der ja der Begriff eine zentrale Rolle spielt.

Besonders diese zweite, Kuhn'sche Verwendungsweise scheint aufgrund ihrer gewichtigen Implikationen interessant und einer kritischen Überprüfung wert zu sein. Gibt es tatsächlich mehrere verschiedene wissenschaftliche Paradigmen innerhalb der Historischen Sprachwissenschaft? Haben sich in den vergangenen Jahrzehnten tatsächlich wissenschaftliche Revolutionen innerhalb des Fachs abgespielt? Haben die neuen Paradigmen tatsächlich die alten abgelöst, wie es nach Kuhn bei wissenschaftlichen Revolutionen geschieht? Oder handelt es sich vielleicht eher nur um eine unreflektierte, vulgarisierende Begriffsverwendung, die vor allem Legitimationszwecken dient? Diese Vermutung liegt nahe, und zwar aus mehreren Gründen.

Erstens ist es bemerkenswert, dass (auch) in der Historischen Sprachwissenschaft auffallend häufig von Paradigmen die Rede ist, ohne dass durch entsprechende wissenschaftsgeschichtliche Analysen auch nur in einem einzigen Fall und auch nur annähernd plausibel nachgewiesen worden wäre, dass die als Paradigmen etikettierten Theorien wirklich über diejenigen Eigenschaften verfügen, über die sie verfügen müssen, wenn es sich bei ihnen um wissenschaftliche Paradigmen handelt. Zweitens haben wissenschaftsgeschichtliche Analysen der vergangenen Jahrzehnte mehrfach und überzeugend gezeigt, dass die Kriterien für wissenschaftliche Revolutionen bzw. Paradigmen im Kuhn'schen Sinne nicht einmal beim linguistischen Strukturalismus und der Generativen Grammatik erfüllt sind (vgl. z. B. Kertész/Rákosi/Bódog 2006, Percival 1976, 1977, 1981, Newmeyer 1986 etc.). Und wenn selbst diese - zweifelsfrei einmalig einflussreichen - Schulen nicht als Paradigmen ausgewiesen werden können, so ist es zumindest fraglich, inwiefern innerhalb der Historischen Sprachwissenschaft von Paradigmen und Revolutionen die Rede sein kann.

Allem Anschein nach haben wir es also auch innerhalb der Historischen Sprachwissenschaft zunächst einfach damit zu tun, was - in unterschiedlichen Kontexten - auch von Fehér (1984: 299), Kertész (2010: 151f.) oder Lakoff (1989: 966) konstatiert wird. Damit nämlich, dass der Terminus ,Paradigma‘ aus soziologischen Gründen, nämlich zu Legitimationszwecken verwendet wird, um die (vermeintliche oder wirkliche) Originalität einer Theorie betonen und die Reife der Disziplin unter Beweis stellen zu können. Disziplinen, die über keine wissenschaftlichen Paradigmen verfügen, werden ja von Kuhn als wissenschaftlich unreif eingestuft 
- und Kuhn selbst ordnet die meisten Sozial- und Geisteswissenschaften tatsächlich als unreif ein (vgl. Kuhn 1976: 30, 35).

Kuhns Wissenschaftstheorie ist - wie oben bereits angedeutet - zur Interpretation von anderen Segmenten der Wissenschaftsgeschichte der Linguistik bereits angewendet worden. Für die Historische Sprachwissenschaft wurde sie jedoch bislang noch nicht fruchtbar gemacht. Eine philologisch gestützte wissenschaftsgeschichtliche Analyse der jüngeren Entwicklungen innerhalb der Disziplin im Lichte der Kuhn'schen Paradigmenlehre wäre jedoch aus mehrfacher Hinsicht ein lohnendes Unterfangen. Zum einen natürlich insofern, als eine solche Analyse zu einem differenzierteren Bild über Natur und Hintergründe des Erkenntnisfortschritts innerhalb der Historischen Sprachwissenschaft verhelfen könnte. Und zum anderen auch insofern, als auf diese Weise erneut getestet werden könnte, ob Kuhns Wissenschaftstheorie, die ja am Beispiel der Naturwissenschaften ausgearbeitet wurde, auf die Linguistik überhaupt anwendbar ist.

Eine solche Analyse kann hier natürlich nicht geleistet werden. Im Folgenden soll es dennoch versucht werden, Kuhns wissenschaftstheoretischen Ansatz auf die Historische Sprachwissenschaft zu projizieren, um wenigstens manche Besonderheiten in der Forschungsentwicklung der letzten Jahrzehnte beleuchten zu können.

\section{Besonderheiten der Forschungsentwicklung}

Nach Kuhn ist für die normalwissenschaftliche Phase einer reifen Wissenschaft die Existenz und Akzeptanz eines einzigen vorherrschenden Paradigmas kennzeichnend. Dieses Paradigma wird von der wissenschaftlichen Gemeinschaft allgemein anerkannt und befolgt. Auf die Historische Sprachwissenschaft bezogen könnte daraus - im Lichte der Ausführungen in den vorangehenden Abschnitten - Mehrfaches folgen. Zum einen, dass sich die Disziplin immer noch in einer unreifen, vorparadigmatischen Phase befindet. Dafür spricht jedenfalls die gegenwärtige Grundlageninstabilität innerhalb des Fachs: Statt einer einzigen vorherrschenden Theorie haben wir es mit einer Vielzahl von konkurrierenden und oft auch inkompatiblen Ansichten über die theoretischen und methodologischen Grundlagen der Forschung zu tun. Es gibt keine allgemein gültigen und von allen geteilten Ansichten darüber, was und wie beschrieben und erklärt werden sollte. Es gibt also keine einhellig akzeptierten methodologischen und theoretischen Standards, so dass es mehrfach und immer wieder vorkommt, dass einzelne Forscher das Fachgebiet von Grund auf neu zu entwickeln versuchen (vgl. Kuhn 1976: 28). Verschiedene Forscher 
untersuchen zum Teil grundverschiedene Aspekte des Gegenstandsbereichs und liefern zum Teil Beschreibungen und Erklärungen, die nicht zusammengeführt bzw. aufeinander bezogen werden können (man vgl. etwa die Resultate der diachronen Sprachtypologie mit denen der Historischen Diskursanalyse, so etwa die Beiträge von Renata Szczepaniak und Noah Bubenhofer/Juliane Schröter in diesem Band). Und da dieser Pluralismus bzw. diese Grundlageninstabilität infolge der Entwicklungen der vergangenen Jahrzehnte nur zugenommen hat, könnte man sogar zum Schluss gelangen, dass die Historische Sprachwissenschaft noch nie so weit vom Zustand der Reife entfernt war, wie sie es heute ist.

Ebenfalls als Zeichen der Unreife könnte der Umstand gedeutet werden, dass es gegenwärtig keine Sprachtheorie und auch keine Forschungsmethodologie innerhalb der Historischen Sprachwissenschaft gibt, die allein vorherrschend wäre und nach ihrem Aufkommen die anderen, bis dahin herrschenden Ansichten verdrängt und abgelöst hätte. Hätte die Historische Sprachwissenschaft den Zustand der Reife erreicht und jemals ein Paradigma erworben, so hätte dies zur Folge haben müssen, dass die konkurrierenden Ansichten mit der Übernahme des neuen Paradigmas allmählich untergehen. Ein wichtiges Kennzeichen für dieses reife Stadium müsste weiters sein, dass fundamentale Neuerungen unterdrückt werden, da sie die Grundposition des Paradigmas und seiner Vertreter erschüttern könnten (Kuhn 1976: 20). All dies ist aber im Falle der Historischen Sprachwissenschaft - wie auch die Beiträge des vorliegenden Bandes eindrucksvoll zeigen - nicht gegeben. Die gegenwärtige Forschungssituation ist zwar von einem Konkurrenzkampf zwischen zahlreichen Ansätzen gekennzeichnet. Doch kann man - trotz der oft deutlich erkennbaren gegenseitigen Abneigungen - nicht behaupten, dass sie einander feindlich gegenüberstehen würden. Abweichende Positionen werden wenn schon nicht begrüßt, zumindest gegenseitig toleriert, so dass es gegenwärtig wohl kaum möglich wäre, einen Ansatz zu identifizieren, der als möglicher Paradigmakandidat zu Lasten der anderen allmählich die Oberhand gewinnen würde.

Ähnlich problematisch wäre die Identifizierung von paradigmatischen Werken in der Geschichte der Disziplin. Wissenschaftliche Revolutionen bzw. Paradigmen sind nämlich nach Kuhn immer an bestimmte Werke gebunden, die „für nachfolgende Generationen von Fachleuten die anerkannten Probleme und Methoden eines Forschungsgebiets [...] bestimmen“ (Kuhn 1976: 25). Sie verändern die Disziplin grundsätzlich und dienen zugleich als Vorbilder, ,aus denen fest gefügte Traditionen wissenschaftlicher Forschung erwachsen“" (ebd.). Selbst wenn natürlich mehrere sehr einflussreiche Leistungen in der Geschichte der Historischen Sprachwissenschaft genannt werden könnten, ist es zu bezweifeln, dass es jemals 
ein allgemein anerkanntes, vorbildhaftes Werk unter ihnen gegeben hat, das die Disziplin - in der Art wie dies etwa Newtons Principia in der Physik getan hat (vgl. ebd.) - grundlegend umgestaltet hätte. Nicht einmal für Hermann Pauls Prinzipien der Sprachgeschichte (Paul 1995) lässt sich dies behaupten, obwohl es nach seinem Erscheinen im Jahre 1880 längere Zeit hindurch tatsächlich als kanonisches Werk galt. Zum einen darf nicht übersehen werden, dass es keineswegs die erste bedeutende Arbeit der Junggrammatiker war. Bereits in der zweiten Hälfte der 1870er Jahre sind grundlegende junggrammatische Arbeiten u. a. von Brugmann, Osthoff, Leskien und Sievers entstanden (vgl. z. B. Leskien 1876, Sievers 1876). Zum anderen kann von einer fundamentalen Veränderung der Disziplin auch insofern nicht die Rede sein, als die Junggrammatiker sowohl von ihrem Untersuchungsgegenstand als auch von ihrer naturwissenschaftlichen Orientierung her in der Tradition der historisch-vergleichenden Sprachwissenschaft standen (vgl. Gardt 1999: 278ff.).

Auf der Suche nach einer Antwort auf die Frage, ob die Historische Sprachwissenschaft den Zustand der Reife im Kuhn'schen Sinne erlangt hat, ist es schließlich auch aufschlussreich, einen Blick auf die gängigen Lehrbücher des Fachs zu werfen. Hat nämlich eine Disziplin den Zustand der Reife erreicht und ein Paradigma erworben, so wird dieses Paradigma - d. h. das anerkannte Theoriegebäude zusammen mit erfolgreichen Anwendungen - zur Ausbildung der zukünftigen Forschergenerationen in Lehrbüchern dargestellt (vgl. Kuhn 1976: 25). Nun sind aber die Einführungen in die Historische Sprachwissenschaft von ihrem Inhalt her alles andere als einheitlich. Vielmehr spiegeln sie die oben beschriebene Grundlageninstabilität wider, die für die Disziplin in ihrem gegenwärtigen $\mathrm{Zu}-$ stand charakteristisch ist. Vergleicht man etwa Schmidt (2007), Polenz (2009) oder Nübling et al. (2010), so wird man schnell feststellen können, dass sie jeweils unterschiedliche Bilder über die Disziplin zeigen - sowohl hinsichtlich des Forschungsgegenstandes als auch in Bezug auf die Methodologie der Forschung wie auch hinsichtlich des Erklärungsanspruchs und des präferierten Erklärungstyps. In diesem Sinne kann man also feststellen, dass die gegenwärtigen Lehrbücher der Historischen Sprachwissenschaft die zukünftigen Forschergenerationen in jeweils unterschiedliche Forschungspraxen einführen - eine Situation, die für reife Wissenschaften eher als untypisch zu gelten hat.

Aber selbst wenn die heutige Situation an vielen Punkten die Merkmale von unreifen Wissenschaften, d. h. von vorparadigmatischen Forschungsphasen trägt, kann man dennoch keineswegs behaupten, dass die Historische Sprachwissenschaft - anderen Bereichen der Linguistik ähnlich (vgl. Kertész/Rákosi/Bódog 2006: 438) - eine unreife Disziplin im 
Kuhn'schen Sinne wäre. Dagegen sprechen jedenfalls mehrere Argumente, von denen hier nur einige angedeutet werden können.

Zum einen hat die Historische Sprachwissenschaft eine relativ hohe Stufe der Professionalisierung erreicht. Die Forschungen werden immer esoterischer, so dass der Laie in den meisten Fällen nicht mehr hoffen darf, „den Fortschritt durch das Lesen der Originalberichte der Fachleute verfolgen zu können“" (Kuhn 1976: 35). Zum anderen sind die Grundlagen der einzelnen etablierten Forschungsrichtungen in Hand- und Lehrbüchern dargestellt, so dass sich die Forscher auf subtile und esoterische Aspekte der untersuchten Phänomene konzentrieren können und nicht gezwungen sind, in Ermangelung von Konventionen ihr Fach jedes Mal von Grund auf neu zu entwickeln (ebd. 28, 34). Dies hat zur Folge, dass das Buch als Publikationsform immer mehr auf Hand- und Lehrbücher beschränkt ist, während die Forschungsresultate zu den einzelnen relevanten Teilproblemen - wie in reifen Wissenschaften üblich - zumeist in Form von kürzeren Aufsätzen in Zeitschriften und Sammelbänden erscheinen. Forschungsmonographien gehen in den meisten Fällen auch in der Historischen Sprachwissenschaft auf Qualifikationsschriften zurück, bei denen die Buchform jedoch konventionell festgelegt ist und erwartet wird. Die Bedeutung von Aufsätzen nimmt aber - wie auch in anderen Bereichen der Linguistik (vgl. Kabatek 2009: 46f.) - generell und immer mehr zu. Drittens ist die Historische Sprachwissenschaft institutionell sehr stark und fest eingebettet: Es sind ihr eigene Lehrstühle gewidmet, sie hat ihre eigenen internationalen Publikationsforen und Fachvereinigungen und das Fach ist auch in universitären Lehrplänen fest verankert. Ein solcher Institutionalisierungsgrad ist jedoch nach Kuhn (1976: 33f.) nur bei Wissenschaften erwartbar, die ihr erstes Paradigma bereits erworben und somit den Zustand der Reife erreicht haben. Und schließlich scheint unter den von Kuhn genannten Reifekriterien auch der Umgang mit linguistischen Daten einer kurzen Reflexion wert zu sein. Kuhn schreibt diesbezüglich Folgendes:

Beim Fehlen eines Paradigmas oder eines Kandidaten für ein Paradigma scheinen alle Tatsachen [...] gleichermaßen relevant zu sein. Folglich ist das Zusammentragen von Fakten in der Frühzeit eine Tätigkeit, die weit mehr dem Zufall unterliegt als die, welche die darauf folgende wissenschaftliche Entwicklung kennzeichnet. (Kuhn 1976: 30)

Nun können wir aber keineswegs behaupten, dass innerhalb der Historischen Sprachwissenschaft ein unreflektierter und undifferenzierter Umgang mit linguistischen Daten herrschen würde. Im Gegenteil scheinen die methodologischen und empirischen Standards immer strenger - wenn auch keineswegs unbedingt einheitlich - zu sein. Die Repräsentativität der 
Daten und die empirische Adäquatheit der Beschreibungen und Erklärungen wird stärker denn je gefordert und reflektiert, wie dies etwa die Beiträge in Lobenstein-Reichmann/Reichmann (2003) oder auch die Texte von Stephan Elspaß, Anna Molnár, Damaris Nübling und Richard J. Watts in diesem Band eindeutig zeigen. Dieser bewusste und reflektierte Umgang mit linguistischen Daten ist zweifelsfrei im Kontext des Aufschwungs der Korpuslinguistik sowie als Folge bzw. als Teil derjenigen intensiven Auseinandersetzung mit der Beschaffenheit linguistischer Daten und Evidenz zu sehen, die innerhalb der Linguistik (vgl. Kertész/Rákosi 2008) und dabei auch innerhalb der Historischen Sprachwissenschaft (vgl. Fischer 2004, Jacobs/Jucker 1995, Lehmann 2004, Nagy C. 2008 etc.) in den vergangenen Jahren stattgefunden hat.

Im Sinne des Gesagten scheinen also die Merkmale der gegenwärtig durch eine ausgeprägte Grundlageninstabilität gekennzeichneten Historischen Sprachwissenschaft weder mit der Kuhn'schen Konzeption von reifen Disziplinen vereinbar zu sein, noch erfüllen sie die Kriterien von unreifen Wissenschaften. Der Ausweg aus diesem Dilemma kann - im Einklang mit Percival (1976), Oesterreicher (1979: 53f.) und Kertész/Rákosi/Bódog (2006) - die Einsicht sein, dass Kuhns Modell, seine Unterscheidung zwischen reifen und unreifen Wissenschaften, viel zu starr ist, als dass sie auf die Linguistik und darin auf die Historische Sprachwissenschaft anwendbar wäre. Dieser Befund steht zwar mit Kuhns eigener Charakterisierung der Sozial- bzw. Geisteswissenschaften als unreife Disziplinen im Widerspruch. Doch er ist insofern nicht unbedingt verwunderlich, als Kuhns Augenmerk ausschließlich auf die Naturwissenschaften gerichtet war. In seinem Buch hat er weder die Linguistik, noch andere geistesbzw. sozialwissenschaftliche Disziplinen eingehend analysiert, so dass sein Werk letztlich die Frage offen ließ, ob die Lehre von der Struktur wissenschaftlicher Revolutionen auf diese Wissenschaften überhaupt angewendet werden kann.

Von den Eigenheiten, die auf die Unvereinbarkeit der Kuhn'schen Lehre mit der internen Dynamik der Historischen Sprachwissenschaft hindeuten, soll hier zum Schluss noch eine weitere kurz angedeutet werden. Es handelt sich um die Frage, durch welche Faktoren die Entstehung von grundlegenden Neuerungen, d. h. die Begründung und Etablierung von neuen Forschungsansätzen in der Disziplin ausgelöst wurde bzw. wird. Dies ist ein Punkt, an dem eine weitere Besonderheit in der jüngeren Forschungsentwicklung erkennbar wird.

Kuhn vertritt bezüglich dieser Frage die Grundthese, dass das Auftreten von konkurrierenden Forschungsweisen, die die Grundlagen der bis dahin herrschenden Forschungspraxis in Frage stellen, letztlich auf Anomalien zurückgeführt werden kann. Damit sind Entdeckungen oder neu- 
artige Tatsachen gemeint, die im Rahmen der alltäglichen Forschungspraxis auftauchen, die aber mit den Mitteln, die die wissenschaftliche Gemeinschaft als adäquat anerkennt und benutzt, nicht beschrieben und erklärt werden können. In dem Maße, wie die Zahl solcher Anomalien wächst und der Misserfolg der Versuche zur Behebung dieser Anomalien bewusst wird, kommt es zur Entstehung und Verbreitung eines Krisenbewusstseins innerhalb der wissenschaftlichen Gemeinschaft. Das Vertrauen zu den bis dahin kollektiv anerkannten Überzeugungen, Methoden und Begriffen schwindet immer mehr (vgl. Kuhn 1976: 11, 65ff.). In diesem Klima kommt es dann zur Formulierung von Neuansätzen, die, wenn sie Erfolg versprechend und neuartig genug sind, auch von anderen Mitgliedern der Gemeinschaft übernommen werden.

Wirft man einen Blick auf die Historische Sprachwissenschaft, so wird man recht schnell feststellen müssen, dass diese Beschreibung bestenfalls zum geringeren Teil auf die Entwicklungen innerhalb der Disziplin zutrifft. Fundamentale Neuerungen, d. h. voneinander relativ unabhängige und inkompatible Ansätze gibt es und gab es - wie gesagt - nicht wenige. Doch solche unter ihnen, deren Entstehung durch solche Anomalien in der alltäglichen Forschungspraxis ausgelöst worden wäre, können kaum genannt werden. Ein Beispiel hierfür könnte immerhin der von Vimos Agel jüngst vorgeschlagene Ansatz einer dynamischen Grammatik (vgl. Ágel 2001, 2003, 2005) sein. Dessen Entwurf wurde - wie in Ágel (2003: 1) berichtet wird - in direkter Weise durch die Absicht motiviert, eine konkrete Forschungsaufgabe, nämlich die Erarbeitung einer neuhochdeutschen Sprachstufengrammatik, zu lösen:

\begin{abstract}
Als künftiger Autor der „Neuhochdeutschen Grammatik“ in der „Sammlung kurzer Grammatiken germanischer Dialekte“ ist man doppelt in die Zange genommen. Denn einerseits hat man die Verpflichtung und auch den Willen, eine ehrwürdige Tradition historischer Grammatikforschung würdig fortzusetzen. Andererseits muss man auch den theoretischen Anforderungen genügen, die an Gegenwartsgrammatiken gestellt werden. Schließlich mündet ja die eigene historische Beschreibung unmittelbar in die Beschreibung der Gegenwartssprache. (Ágel 2003: 1)
\end{abstract}

Hier handelt es sich also tatsächlich um ein Forschungsproblem, das sich in eine Reihe von weiteren Problemen fügt, die sich im Rahmen einer anerkannten Forschungstradition stellen und von der wissenschaftlichen Gemeinschaft als relevant anerkannt werden. Bei der Lösung dieses Problems traten aber Anomalien, d. h. in diesem Forschungsrahmen nicht zu lösende Probleme auf, die letztlich zur Ablehnung der bis dahin befolgten Forschungsnormen und zur Erarbeitung von radikal abweichenden Forschungsgrundlagen führten (vgl. dazu auch Molnár, in diesem Band). 
Doch dieser Weg scheint keineswegs der übliche zu sein. Vielmehr werden neue, konkurrierende Sichtweisen - besonders in der jüngeren Forschungsgeschichte - zumeist von außen, durch den interdisziplinären Austausch mit anderen linguistischen und extralinguistischen Disziplinen, herangetragen. Es sind also weit weniger die im Rahmen der gängigen Forschungspraxis auftauchenden Anomalien als vielmehr soziologische Motive und nicht zuletzt natürlich die persönlichen Interessen der Forscher, die die Entwicklungsdynamik innerhalb der Forschung prägen. Als herausgegriffenes Beispiel hierfür soll an dieser Stelle der Entstehungskontext von lediglich zwei jüngeren Ansätzen, nämlich der Pragmatischen Sprachgeschichte (vgl. z. B. Sitta 1980, Cherubim 1998), sowie der Europäischen Sprachgeschichte (vgl. z. B. Reichmann 2001, 2002, Mattheier 2010) kurz erwähnt werden.

Die Forderung zur Pragmatisierung der Sprachgeschichtsschreibung ist innerhalb der Germanistik ab den 1980er Jahren immer häufiger und prägnanter formuliert worden. Doch den Anlass dazu gab nicht etwa das Versagen der bis dahin weitgehend vorherrschenden philologisch-junggrammatischen Forschungstradition. In diesem Rahmen wird ja auch bis heute weiter gearbeitet. Vielmehr ging es - wie in Abschnitt 3 ausgeführt darum, durch den Anschluss an progressive Forschungsrichtungen außerhalb des Fachs, in diesem Fall eben an die Pragmatik, die Historische Sprachwissenschaft zu erneuern und auch in den Augen der linguistischen Außenwelt wieder salonfähig zu machen. Hierzu ein Zitat aus einer Podiumsdiskussion, die unter dem bezeichnenden Titel Was soll Gegenstand der Sprachgeschichtsforschung sein? im Rahmen eines Symposions im Jahre 1992 veranstaltet wurde:

Systemgeschichte soll zum Gesellschaftlichen hin funktionalisiert werden, um zu vermeiden, daß lediglich lautliche oder morphologische Systeme „rekonstruiert“ bzw. „hypostasiert“" werden, „die mit dem Handeln von Menschen oft herzlich wenig zu tun haben“ (D. Cherubim). (Reichmann et al. 1995: 455)

Am Zitat wird deutlich erkennbar, dass hinter der Forderung der Pragmatisierung in Wirklichkeit nichts anderes als die Absicht des Bruchs mit der junggrammatischen Tradition steckt. Die Argumentation erfolgt bereits aus einer genuin pragmatischen Perspektive, aus der die junggrammatischen Forschungskonventionen natürlich als defizitär erscheinen. Die Pragmatisierung der Sprachgeschichtsforschung wird nicht deswegen befürwortet, weil sie die Beseitigung von Anomalien verspricht, die im Rahmen der früheren Forschung nicht aus dem Weg geräumt werden konnten. Pragmatische Fragestellungen wurden ja bis dahin gar nicht erst formuliert. Die pragmatische Perspektive wird stattdessen insofern als nütz- 
lich dargestellt, als durch sie der viel getadelte junggrammatische Atomismus überwunden werden kann.

In Bezug auf das Konzept der Europäischen Sprachgeschichte tritt uns im folgenden Zitat eine dezidiert soziologisch orientierte Argumentation entgegen. Auch in diesem Fall wird von dem neuen Ansatz nicht die Lösung von bis dahin als unlösbar erscheinenden Problemen erwartet. Im Zentrum der Argumentation steht die - viel zu oft außer Acht gelassene Erkenntnis, dass auch wissenschaftliche Forschung im sozialen Kontext stattfindet und gesellschaftliche Interessen bedienen muss:

(1) Wenn sich vor allem in der Schule, aber auch an der Universität auf die Dauer nichts $[\ldots]$ hält, was nicht in der Gesellschaft als relevant, wichtig, interessant befunden wird, dann verliert die Sprachgeschichtsforschung herkömmlicher einzelsprachbezogen-nationaler Ausrichtung in dem Maße ihre gesellschaftliche Begründung, wie sich eine neue [...] Identität ,Europa' [...] bildet. (2) Wenn man dem dadurch zu entgehen versucht, dass man die einzelsprachbezogen-nationale Sprachgeschichtsschreibung ihrer nationalen Komponenten entkleidet, [...] dann ergibt sich vermutlich ein gesellschaftliches Motivationsproblem. Eine maximal sachlich (was auch immer das heißen mag) aufgezogene Geschichte einer Einzelsprache könnte sich als ohne Identifikationspotential entpuppen [...]. (3) Man wird also [...] eine Neubegründung der Sprachgeschichtsforschung in dem Sinne vorzunehmen haben, dass die Einzelsprache aus ihrem europäischen Rahmen heraus beschrieben wird. (Reichmann 2002: 40)

Und last but not least wird die Unvereinbarkeit des Kuhn'schen Wissenschaftsbildes mit der Historischen Sprachwissenschaft auch am Beispiel der in den vergangenen Jahrzehnten wieder sehr intensiv betriebenen Forschungen zur Sprachwandeltheorie erkennbar (vgl. Coseriu 1974, Croft 2000, Keller 1990, Labov 1994, 2001, 2010, Wurzel 1988 etc.). Man kann ja nicht behaupten, dass die Entstehung dieser neueren Sprachwandeltheorien von neuen Daten und Entdeckungen herbeigeführt worden wäre, die frühere Theorien falsifiziert hätten. Umso weniger ist dies der Fall, als die meisten modernen Sprachwandeltheorien von Forschern aufgestellt worden sind, die bis dahin zumeist oder ausschließlich außerhalb der Historischen Sprachwissenschaft tätig waren. Freilich gibt es auch Ausnahmen (vgl. etwa Lass 1980), doch insgesamt kann nach Hermann Paul kaum ein anderer führender Sprachwandeltheoretiker genannt werden, der selbst mit historischen Daten gearbeitet hätte (oder umgekehrt kaum ein Sprachhistoriker, der mit einer Sprachwandeltheorie hervorgetreten wäre). Die wichtigsten Impulse für diese Theorien lieferten also weniger in Anomalien wurzelnde Krisen als vielmehr Probleme und Erkenntnisse anderer linguistischer und extralinguistischer Disziplinen wie z. B. der Soziologie bzw. der Soziolinguistik (Labov, Croft), der Evolutionsbiologie (Croft) oder der Wirtschaftswissenschaft (Keller). 
Zusammengefasst kann also festgestellt werden, dass Kuhns Wissenschaftstheorie allem Anschein nach nicht in der Lage ist, all diejenigen Entwicklungen zu erklären, die sich im Laufe der Geschichte der Historischen Sprachwissenschaft abgespielt haben. Aber auch - und ganz besonders - die heutige Situation der Historischen Sprachwissenschaft zeichnet sich durch Eigenheiten aus, die sich vom von Kuhn gezeichneten Bild der Naturwissenschaften aber auch vom früheren Profil der Disziplin an vielen Punkten grundsätzlich unterscheiden. Wohin die geschilderten Entwicklungen der vergangenen Jahrzehnte führen werden, lässt sich kaum voraussagen. Auf der einen Seite hat es den Anschein, dass die Diversifikation und auf diese Weise die Grundlageninstabilität innerhalb des Fachs immer mehr zunimmt, da ältere Ansätze neben den neueren stets bestehen bleiben. Auf der anderen Seite führt aber der interdisziplinäre Austausch zur gleichen Zeit auch zu theoretischen und methodologischen Annäherungen und Konvergenzen, sowohl zwischen einzelnen Forschungsrichtungen innerhalb der Disziplin als auch zwischen der Historischen Sprachwissenschaft und anderen Disziplinen innerhalb wie außerhalb der Linguistik. Diese intensive und vielfältige Dynamik der letzten Jahrzehnte deutet zum einen auf eine in der Geschichte des Fachs wohl nie vorher vorhandene Vitalität hin. Zum anderen zeugt sie davon, dass die Historische Sprachwissenschaft ihre einstige periphere Stellung innerhalb der Linguistik verlassen hat und heute eindeutig zu den progressiven und innovativen Disziplinen innerhalb der Linguistik gehört.

\section{Zu den Beiträgen des Bandes}

All diese Entwicklungen und Merkmale werden auch von den Beiträgen dieses Sammelbandes widerspiegelt. Sie vermögen zwar kein repräsentatives Bild über sämtliche Gegenstände, Methoden und Theorien innerhalb des Fachs zu geben. Durch ihre zumeist selbstreflexive Ausrichtung und ihre kritische Perspektive zeigen sie aber zahlreiche zentrale Entwicklungstendenzen, Desiderate und Zukunftsperspektiven des Fachs auf. Sie vertreten die verschiedensten Forschungsrichtungen: die Sprachwandeltheorie, die Diachrone Sprachtypologie und die Historische Grammatikographie; weiters die Grammatikalisierungsforschung, die Historisch-vergleichende Sprachwissenschaft, die Sprachgeschichtsschreibung, dabei u. a. die kulturhistorisch orientierte Sprachgeschichte sowie - innerhalb der Soziopragmatischen Sprachgeschichte - die Sprachgebrauchsgeschichte, die Sprachbewusstseinsgeschichte und die Historische Pragmatik. Damit decken sie zwar keineswegs das gesamte Forschungsspektrum innerhalb des Fachs ab, auf jeden Fall wird aber an ihnen diejenige Vielfalt an 
Gegenständen, Methoden und Theorien erkennbar, die für die Disziplin heute charakteristisch ist. Die meisten Beiträge sind aus einer germanistischen Perspektive heraus entstanden. Je ein Beitrag gewährt aber auch einen Einblick in einschlägige Probleme und Fragestellungen innerhalb der Anglistik (Richard J. Watts) und der Finnougristik (Marianne BakróNagy).

An den Fragestellungen, methodologischen Herangehensweisen und theoretischen Orientierungen der einzelnen Aufsätze werden auch die vielfältigen interdisziplinären Beziehungen sichtbar, die die Historische Sprachwissenschaft von heute maßgeblich prägen. Ohne Vollständigkeitsanspruch kann in diesem Zusammenhang unter den linguistischen Teildisziplinen vor allem die Soziolinguistik (u. a. Elspaß, Takada, Watts), die Korpuslinguistik (Bubenhofer/Scharloth, Bubenhofer/Schröter, Elspaß), die Kognitive Linguistik (Watts, Kiss) oder die Sprachtypologie (Nübling, Szczepaniak) genannt werden; von den Wissenschaftsbereichen außerhalb der Linguistik wiederum u. a. die Kulturwissenschaft (Gardt), die Biologie (Kiss), die Mentalitätsgeschichte oder die discourse studies (Bubenhofer/ Schröter).

In mehreren Beiträgen wird für den Abbau von (Teil)Disziplinengrenzen und die gegenseitige Annäherung von einst relativ autonomen und einander kaum wahrnehmenden linguistischen Forschungsgebieten argumentiert. So plädiert etwa Anna Molnár für mehr Austausch und gegenseitige Offenheit zwischen Grammatikalisierungsforschung und Historischer Grammatikographie; Renata Szczepaniak für die Anwendung sprachtypologischer Beschreibungs- und Erklärungsinstrumentarien in der Historischen Phonologie; Marianne Bakró-Nagy für die Einbeziehung von Erkenntnissen der Allgemeinen Sprachwissenschaft (u. a. der Sprachtypologie, der Grammatikalisierungsforschung oder der Laborphonologie) auch in etymologischen Forschungen; und Noah Bubenhofer/Joachim Scharloth für den Anschluss der (historischen) Stilanalyse an Methoden der Korpuspragmatik.

Auf der anderen Seite wird man aber an den Fragestellungen, methodologischen Reflexionen und nicht zuletzt natürlich an den kritischen, explizit oder implizit wertenden Äußerungen der Autoren auch die Konkurrenzen, Inkompatibilitäten und gegenseitige Abneigungen erkennen können, die - nicht selten unüberwindbar erscheinende - Trennlinien zwischen den einzelnen Forschungsrichtungen signalisieren. Auf der einen Seite plädiert etwa Damaris Nübling aus einer typologischen Perspektive heraus - ähnlich zu Anna Molnár - für problemorientiertes Denken und somit gegen eine isolierte Behandlung der einzelnen sprachhistorischen Epochen. Auf der anderen Seite geht Paul Rössler gerade der Frage nach, wie eine möglichst empirisch adäquate und sprachgeschichtsdidaktisch 
vorteilhafte Periodisierung der deutschen Sprachgeschichte aussehen könnte. In ähnlicher Weise argumentiert Stephan Elspaß - im Einklang mit Dieter Cherubim, Jenő Kiss und Richard J. Watts - dagegen, in sterilen, in sich geschlossenen Sprachsystemen zu denken und bei Sprachwandelerklärungen den sozialen Kontext, d. h. die außersprachlichen Bedingungsfaktoren des Sprachgebrauchs gänzlich auszublenden. Auf der anderen Seite spricht sich aber Damaris Nübling entschieden für eine sprachtypologische Orientierung und damit für systemimmanente und prinzipiengesteuerte Erklärungen aus, da diese Richtung ihrer Ansicht nach ,weit mehr verspricht als nur nach Sprachkontaktszenarien oder dem Einfluss gesellschaftlicher Veränderungen auf die Sprache zu suchen". Und während sie sich - zusammen mit Renata Szczepaniak - für eine sprachtypologisch orientierte Historische Linguistik einsetzt und deren Nutzen an mehreren Beispielen auch veranschaulicht, widmet sich Stephan Elspaß in seinem Beitrag u. a. gerade auch der Frage, ob und inwieweit die zwangsläufigen Idealisierungen und Abstraktionen in der typologischen Arbeitsweise vertretbar und die zugrunde gelegten Daten im Sinne von Ágel (2001: 139) - empirisch viabel seien. ${ }^{8}$

Generell lässt sich sagen, dass in den meisten Beiträgen des Bandes verschiedene Elemente der gegenwärtigen Forschungspraxis kritisch reflektiert und nicht selten sogar grundsätzlich in Frage gestellt werden. So kritisiert etwa Richard J. Watts den gegenwärtig immer noch viel zu verengten, selektiven und teleologischen Blick auf die Sprachgeschichte, der zu einem bedeutenden Teil der metaphorischen Struktur des wissenschaftlichen Diskurses bzw. einer Reihe von ideologisch generierten und diskursiv verfestigten Sprachmythen verschuldet ist. Die Beiträge von Noah Bubenhofer/Juliane Schröter, Noah Bubenhofer/Joachim Scharloth und Stephan Elspaß können u. a. auch als ein Plädoyer für eine korpuslinguistische Neuorientierung in sprachhistorischen Forschungen gelesen werden. Und Anna Molnár weist in ihrem Aufsatz - im Einklang mit Ágel (2005) - auf die Unzulänglichkeit der Beschreibungskategorien wie auch der Anlage historischer Grammatiken hin, die ihrerseits u. a. die Integration von relevanten Erkenntnissen der Grammatikalisierungsforschung in die Historische Grammatikographie verhindern.

Diese sind nur einige herausgegriffene Kritikpunkte und Forderungen, die neben den vielen anderen auf Schwachstellen bzw. aktuelle und möglicherweise die zukünftige Forschung prägende Entwicklungstendenzen hindeuten. An ihnen wird nicht zuletzt erkennbar, dass der besonders ab den 1980er Jahren einen enormen Aufschwung genommene Erneuerungsprozess innerhalb des Fachs bis heute andauert und keineswegs zu

8 Zu dieser Frage vgl. auch die Ausführungen in Oesterreicher (2001: 1573ff.). 
einem konsenshaften Abschluss gekommen ist. Die Entstehung eines einzigen einheitlichen, von der wissenschaftlichen Gemeinschaft allgemein anerkannten Paradigmas im Kuhn'schen Sinne ist in der näheren Zukunft allem Anschein nach nicht zu erwarten. Dies kann aber nur solange als negatives Zeichen gedeutet werden, solange man verkrampft am Kuhn'schen Wissenschaftsbild festhält und damit stillschweigend auch die Annahme akzeptiert, dass auch die Geisteswissenschaften nach dem von Kuhn über die Naturwissenschaften gezeichneten Bild zu funktionieren haben. Ist dies aber nicht der Fall, so kann die Vielfalt der Erkenntniswege umgekehrt gerade als Vorteil, u. a. als Zeichen der Vitalität und der Innovativität der Disziplin gesehen werden. Auf jeden Fall gilt aber, diesen Pluralismus und diese Grundlageninstabilität sowohl unter epistemologischem als auch unter soziologischem Aspekt zu reflektieren und bewusst zu steuern. Es bleibt zu wünschen, dass die Aufsätze dieses Sammelbandes zu einer solchen bewussten und reflektierten Auseinandersetzung mit den Grundlagen der Forschung anspornen und somit auch zum objektwissenschaftlichen Erkenntnisfortschritt in der Historischen Sprachwissenschaft beitragen mögen.

\section{Literatur}

Ágel, Vilmos (1999): Grammatik und Kulturgeschichte. Die raison graphique am Beispiel der Epistemik. In: Gardt, Andreas/Haß-Zumkehr, Ulrike/Roelcke, Thorsten (Hrsg.): Sprachgeschichte als Kulturgeschichte. Berlin/New York: de Gruyter, 171-223.

Ágel, Vilmos (2001): Gegenwartsgrammatik und Sprachgeschichte. Methodologische Überlegungen am Beispiel der Serialisierung im Verbalkomplex. In: Zeitschrift für germanistische Linguistik 29, 319-331.

Ágel, Vilmos (2003): Prinzipien der Grammatik. In: Lobenstein-Reichmann, Anja/Reichmann, Oskar (Hrsg.), 1-46.

Ágel, Vilmos (2005): Wort-Arten aus Nähe und Distanz. In: Knobloch, Clemens/Schaeder, Burkhard (Hrsg.): Wortarten und Grammatikalisierung. Perspektiven im System und Erwerb. Berlin/New York: de Gruyter, 95-129.

Anttila, Raimo (1975): Revelation as Linguistic Revolution. In: Makkai, Adam/Makkai, Valerie Becker (eds.): The First LACUS Forum 1974. Columbia, South Carolina: Hornbeam Press, 171-176.

Arens, Hans (1974): Sprachwissenschaft. Der Gang ihrer Entwicklung von der Antike bis zur Gegenwart. 2. Auflage. Frankfurt/M.: Athäneum Fischer. 
Bahner, Werner/Neumann, Werner (Hrsg.) (1985): Sprachwissenschaftliche Germanistik. Ihre Herausbildung und Begründung. Berlin: Akademie-Verlag.

Barnes, Barry/Bloor, David/Henry, John (1996): Scientific Knowledge: A Sociological Analysis. London: Athlone Press.

Besch, Werner et al. (Hrsg.) (1998): Sprachgeschichte. Ein Handbuch zur Geschichte der deutschen Sprache und ihrer Erforschung. 2., vollständig neu bearbeitete und erweiterte Auflage, Bd. 1. Berlin/New York: de Gruyter.

Boretzky, Norbert (1977): Einführung in die historische Linguistik. Reinbek bei Hamburg: Rowohlt.

Cherubim, Dieter (1975): Einleitung. In: Cherubim, Dieter (Hrsg.): Sprachwandel. Reader zur diachronen Sprachwissenschaft. Berlin/ New York: de Gruyter, 1-61.

Cherubim, Dieter (1998): Sprachgeschichte im Zeichen der linguistischen Pragmatik. In: Besch, Werner et al. (Hrsg.), 538-550.

Coseriu, Eugenio ([1958]1974): Synchronie, Diachronie und Geschichte. Das Problem des Sprachwandels. München: Fink.

Croft, William (2000): Explaining Language Change: An Evolutionary Approach. Harlow, Essex: Longman.

Fehér, Márta (1984): Thomas Kuhn tudományfilozófiai ,paradigmája [Thomas Kuhns wissenschaftsphilosophisches ,Paradigma '. In: Kuhn, Thomas S.: A tudományos forradalmak szerkezete [Die Struktur wissenschaftlicher Revolutionen]. Budapest: Gondolat, 299-318.

Fischer, Olga (2004): What counts as evidence in historical linguistics? In: Studies in Language 28, 710-740.

Gardt, Andreas (1999): Geschichte der Sprachwissenschaft in Deutschland vom Mittelalter bis ins 20. Jahrhundert. Berlin/New York: de Gruyter.

Gardt, Andreas/Mattheier, Klaus J./Reichmann, Oskar (1995): Vorwort der Herausgeber. In: Gardt, Andreas/Mattheier, Klaus J./Reichmann, Oskar (Hrsg.), VII-X.

Gardt, Andreas/Mattheier, Klaus J./Reichmann, Oskar (Hrsg.) (1995): Sprachgeschichte des Neuhochdeutschen. Gegenstände, Methoden, Theorien. Tübingen: Niemeyer.

Gray, Bennison (1976): Counter-Revolution in the Hierarchy. In: Forum Linguisticum 1, 38-50.

Greule, Albrecht (2005): Emil Skála und die Kanzleisprachenforschung. In: brücken. Germanistisches Jahrbuch Tschechien-Slowakei 2004, $19-28$.

Jacobs Andreas/Jucker, Andreas H. (1995): The historical perspective in pragmatics. In: Jucker, Andreas H. (ed.): Historical Pragmatics: Prag- 
matic Developments in the History of English. Amsterdam/Philadelphia: Benjamins, 3-33.

Jakobson, Roman (1931): Prinzipien der historischen Phonologie. In: Travaux du Cercle Linguistique de Prague 4, 247-267.

Kabatek, Johannes (2009): Publikationsverhalten in der Linguistik. In: Alexander von Humboldt Stiftung (Hrsg.): Publikationsverhalten in unterschiedlichen wissenschaftlichen Disziplinen. Beiträge zur Beurteilung von Forschungsleistungen. 2., erweiterte Auflage. Bonn: Alexander von Humboldt-Stiftung, 46-49.

Keller, Rudi (1990): Sprachwandel. Von der unsichtbaren Hand der Sprache. (UTB; 1567). Tübingen/Basel: Francke.

Kertész, András (2009): Miért érdemes a nyelvésznek reggelente felkelni az ágyból? Avagy: Chomsky „forradalmai“ és a generatív nyelvészet historiográfiája [Warum lohnt es sich für den Linguisten, morgens aus dem Bett zu steigen? Oder: Chomskys „Revolutionen“ und die Historiographie der generativen Linguistik]. 1. Teil. In: Magyar Nyelv 105, 385-401.

Kertész, András (2010): Miért érdemes a nyelvésznek reggelente felkelni az ágyból? Avagy: Chomsky „forradalmai“ és a generatív nyelvészet historiográfiája [Warum lohnt es sich für den Linguisten, morgens aus dem Bett zu steigen? Oder: Chomskys „Revolutionen“ und die Historiographie der generativen Linguistik]. 3. Teil. In: Magyar Nyelv 106, 145-156.

Kertész, András/Rákosi, Csilla (eds.) (2008): New Approaches to Linguistic Evidence. Pilot Studies/Neue Ansätze zu linguistischer Evidenz. Pilotstudien. Frankfurt/M.: Peter Lang.

Kertész, András/Rákosi, Csilla (2008): Daten und Evidenz in linguistischen Theorien: Ein Forschungsüberblick. In: Kertész, András/Rákosi, Csilla (eds.), 21-60.

Kertész András/Rákosi, Csilla/Bódog Alexa (2006): A saussure-i strukturalizmus tudománytörténeti rekonstrukciójáról [Über die wissenschaftsgeschichtliche Rekonstruktion des Saussure'schen Strukturalismus]. In: Magyar Nyelv 102, 430-442.

Kuhn, Thomas S. (1976): Die Struktur wissenschaftlicher Revolutionen. 2., revidierte und um das Postskriptum von 1969 ergänzte Auflage. Frankfurt/M.: Suhrkamp.

Labov, William (1994): Principles of Linguistic Change: Internal Factors. Oxford: Wiley-Blackwell.

Labov, William (2001): Principles of Linguistic Change: Social Factors. Oxford: Wiley-Blackwell.

Labov, William (2010): Principles of Linguistic Change: Cognitive and Cultural Factors. Oxford: Wiley-Blackwell. 
Lakoff, Robin (1989): The way we were; or; The real truth about generative semantics: A memoir. In: Journal of Pragmatics 13, 939-988.

Lass, Roger (1980): On Explaining Language Change. Cambridge: Cambridge University Press.

Lehmann, Christian ([1982]1995): Thoughts on Grammaticalization. München/Newcastle: Lincom Europa.

Lehmann, Christian (2004): Theory and method in grammaticalization. In: Zeitschrift für germanistische Linguistik 32, 152-187.

Leskien, August (1876): Deklination im Slawisch-Litauischen und Germanischen. Leipzig: Hirzel.

Lobenstein-Reichmann, Anja/Reichmann, Oskar (Hrsg.) (2003): Neue historische Grammatiken. Zum Stand der Grammatikschreibung historischer Sprachstufen des Deutschen und anderer Sprachen. Tübingen: Niemeyer.

Maitz, Péter (i. Vorb.): Metasprachgeschichte.

Mattheier, Klaus J. (1995): Sprachgeschichte des Deutschen: Desiderate und Perspektiven. In: Gardt, Andreas/Mattheier, Klaus J./Reichmann, Oskar (Hrsg.), 1-18.

Mattheier, Klaus J. (1998): Allgemeine Aspekte einer Theorie des Sprachwandels. In: Besch, Werner et al. (Hrsg.), 824-836.

Mattheier, Klaus J. (2010): Is there a European language history? In: Multilingua 29, 353-360.

Mayerthaler, Willi (1981): Morphologische Natürlichkeit. Wiesbaden: Athenaion.

Mayerthaler, Willi (1998): Sprachgeschichte in der Sicht der Generativen Transformationsgrammatik. In: Besch, Werner et al. (Hrsg.), 529-538.

Murray, Stephen O. (1980): Gatekeepers and the Chomskyan revolution. In: Journal of the History of the Behavioral Sciences 16, 73-88.

Nagy C., Katalin (2008): Data in historical pragmatics: A case study on the Catalan periphrastic perfective past. In: Kertész, András/Rákosi, Csilla (eds.), 171-197.

Newmeyer, Frederick J. (1986): Has there been a ,Chomskyan revolution“ in linguistics? In: Language 62, 1-18.

Nübling, Damaris et al.: (2010): Historische Sprachwissenschaft des Deutschen. Eine Einführung in die Prinzipien des Sprachwandels. 3., überarbeitete Auflage. Tübingen: Narr.

Oesterreicher, Wulf (1979): Sprachtheorie und Theorie der Sprachwissenschaft. Heidelberg: Winter.

Oesterreicher, Wulf (2001): Historizität - Sprachvariation, Sprachverschiedenheit, Sprachwandel. In: Haspelmath, Martin et al. (eds.): Language Typology and Language Universals. Bd. 2. Berlin/New York: de Gruyter, 1554-1595. 
Paul, Hermann ([1880]1995): Prinzipien der Sprachgeschichte. 10. Auflage. Tübingen: Niemeyer.

Percival, W. Keith (1976): The applicability of Kuhn's paradigms to the history of linguistics. In: Language 52, 285-294.

Percival, W. Keith (1977): Review of E. F. K. Koerner, Ferdinand de Saussure: Origin and Development of his Linguistic Thought in Western Studies of Language. In: Language 53, 383-405.

Percival, W. Keith (1981): The Saussurean paradigm: Fact or fantasy? In: Semiotica 36, 33-49.

Polenz, Peter von (2000): Deutsche Sprachgeschichte vom Spätmittelalter bis zur Gegenwart. Band 1: Einführung. Grundbegriffe. 14. bis 16. Jahrhundert. Berlin/New York: de Gruyter.

Polenz, Peter von (2009): Geschichte der deutschen Sprache. 10., völlig neu bearbeitete Auflage. Berlin/New York: de Gruyter.

Reichmann, Oskar (1998): Sprachgeschichte: Idee und Verwirklichung. In: Besch, Werner et al. (Hrsg.): Sprachgeschichte. Ein Handbuch zur Geschichte der deutschen Sprache und ihrer Erforschung. 2., vollständig neu bearbeitete und erweiterte Auflage. Bd. 1. Berlin/New York: de Gruyter, 1-41.

Reichmann, Oskar (2001): Das nationale und das europäische Modell in der Sprachgeschichtsschreibung des Deutschen. Freiburg: Universitätsverlag Freiburg Schweiz.

Reichmann, Oskar (2002): Nationale und europäische Sprachgeschichtsschreibung. In: Cherubin [sic!], Dieter/Jakob, Karlheinz/Linke, Angelika (Hrsg.): Neue deutsche Sprachgeschichte. Mentalitäts-, kultur- und sozialgeschichtliche Zusammenhänge. Berlin/New York: de Gruyter, 25-42.

Reichmann, Oskar [zus. mit Dieter Cherubim, Johannes Erben, Joachim Schildt, Hugo Steger, Erich Strassner] (1995): Podiumsdiskussion: Was soll der Gegenstand der Sprachgeschichtsforschung sein? In: Gardt, Andreas/Mattheier, Klaus J./Reichmann, Oskar (Hrsg.), 455-459.

Romaine, Susanne (1982): Socio-Historical Linguistics: Its Status and Methodology. Cambridge: Cambridge University Press.

Scharloth, Joachim (2005): Pragmatische Geschichte(n). Theorie der Sprachgeschichte und Erzählstrategien bei Adelung und dem soziopragmatischen Paradigma. In: Beiträge zur Geschichte der Sprachwissenschaft $15,31-52$.

Schmidt, Hartmut (1985): Aspekte der Institutionalisierung. Zur Durchsetzung der neuen Denkmuster. In: Bahner, Werner/Neumann, Werner (Hrsg.), 151-248. 
Schmidt, Jürgen Erich (1988): Saussures Originalität - eine Legende. Der Einfluß der Junggrammatiker auf die Entstehung des Strukturalismus. In: Frankfurter Allgemeine Zeitung 256 (2.11.1988), 3.

Schmidt, Wilhelm (2007): Geschichte der deutschen Sprache. Ein Lehrbuch für das germanistische Studium. 10., verbesserte und erweiterte Auflage. Stuttgart: Hirzel.

Sievers, Eduard (1876): Grundzüge der Lautphysiologie zur Einführung in das Studium der Lautlehre der indogermanischen Sprachen. Leipzig: Breitkopf \& Härtel.

Sitta, Horst (Hrsg.) (1980): Ansätze zu einer pragmatischen Sprachgeschichte. Zürcher Kolloquium 1978. Tübingen: Niemeyer.

Storost, Jürgen (1985): Zeitschriften und Rezensionen. In: Bahner, Werner/Neumann, Werner (Hrsg.), 282-328.

Weinreich, Uriel/Labov, William/Herzog Marvin I. (1968): Empirical foundations for a theory of language change. In: Lehmann, Winfred/ Malkiel, Yakov (eds.): Directions for Historical Linguistics. Austin: University of Texas Press, 95-195.

Wurzel, Wolfgang Ullrich (1984): Flexionsmorphologie und Natürlichkeit. Ein Beitrag zur morphologischen Theoriebildung. Berlin: AkademieVerlag.

Wurzel, Wolfgang Ullrich (1988): Zur Erklärbarkeit sprachlichen Wandels. In: Zeitschrift für Phonetik, Sprachwissenschaft und Kommunikationsforschung 41, 488-510. 
